\title{
Osteopontin Overproduction Is Associated with Progression of Glomerular Fibrosis in a Rat Model of Anti-Glomerular Basement Membrane Glomerulonephritis
}

\author{
Justin Merszei ${ }^{a} \quad$ Jean Wu ${ }^{b}$ Lisa Torres ${ }^{b} \quad$ John M. Hicks ${ }^{c}$ Todd Bartkowiak ${ }^{b}$ \\ Filemon Tan ${ }^{d}$ Ya-Huan Lou ${ }^{b}$ \\ ${ }^{a}$ Department of Medicine, Baylor College of Medicine, ${ }^{b}$ Department of Diagnostic Sciences DB, University of \\ Texas Health Science Center at Houston, 'Texas Children's Hospital, and dDepartment of Internal Medicine, \\ Medical School, University of Texas Health Science Center at Houston, Houston, Tex., USA
}

\section{Key Words}

Fibrosis · Anti-glomerular basement membrane disease $\cdot$

Fibroblast $\cdot$ Extracellular matrix $\cdot$ Inflammation

\begin{abstract}
Background: Glomerular fibrosis is the common end result of glomerulonephritis (GN) regardless of etiology. In our rat model for anti-glomerular basement membrane GN, severe fibrosis follows glomerular inflammation. We investigated the association between expression of extracellular matrix (ECM) proteins and progression of glomerular fibrosis. Methods: Expression of ECM genes in glomeruli was determined at RNA and protein levels. Immunofluorescence was applied to identify cell sources for the molecules. Results: DNA microarray for ECM genes, quantitative RT-PCR and Western blot revealed significant upregulation of osteopontin (OPN), a multifunctional molecule, in the glomeruli only after onset of glomerular fibrosis. Two-dimensional electrophoresis showed that the expressed OPN was in three major isoforms. Immunofluorescence showed that fibrotic tissues in glomeruli accumulated massive deposits of extracellular OPN. Both in vivo and in vitro experiments showed that a novel population of multinucleated $\alpha$-smooth muscle actin ${ }^{+} \mathrm{CD} 90^{-}$
\end{abstract}

\section{KARGER}

(c) 2010 S. Karger AG, Basel

Fax +41613061234 E-Mail karger@karger.ch www.karger.com www.karger.com/ajn myofibroblast-like cells, which surrounded fibrotic tissue, was the main source of OPN during progression of fibrosis. Since senescence-associated $\beta$-galactosidase activity was detected in those cells both in vitro and in vivo, these cells probably were terminally differentiated senescent myofibroblasts. Conclusion: OPN has been implicated in fibrosis in several organs. Our results suggest potential roles of OPN and its main source, the senescent myofibroblasts, in glomerular fibrosis.

Copyright $\odot 2010$ S. Karger AG, Basel

\section{Introduction}

Regardless of etiology, glomerulonephritis (GN) such as anti-glomerular basement membrane (GBM) GN, eventually progresses to glomerular fibrosis, a key step toward end-stage renal disease [1]. Intensive studies in the past decade have shown glomerular fibrosis to be a very complicated process, involving many molecules and cells $[2-5]$. The TGF- $\beta /$ Smad pathway plays a central role in mesangial cell activation and differentiation of fibroblasts, which in turn produce extracellular matrix (ECM) protein. It is less clear how ECM proteins are incorpo-

Dr. Ya-Huan Lou

Department of Diagnostic Science, Dental Branch

University of Texas Houston Health Science Center at Houston

Houston, TX 77030 (USA)

Tel. +1 713500 4059, Fax +1 713500 4500,E-Mail Yahuan.Lou@uth.tmc.EDU 
rated into the architecture of fibrotic tissue and whether those proteins in turn can regulate gene expression in myofibroblasts.

ECM protein osteopontin (OPN) was first identified in bone tissue and participates in calcium deposition during bone generation [6]. OPN is also implicated in a number of physiological and pathological events, including maintenance or re-establishment of tissue integrity, inflammatory processes and tumor metastasis [7-9]. OPN can function as both an anti- and proinflammatory molecule and plays a critical role in autoimmune pathogenesis $[10,11]$. Recently, involvement of OPN in fibrosis in several organs has been demonstrated [12-14]. The OPN mutant mice showed a greater disorganization of matrix in their skin incisions [15]. OPN is required for differentiation of myofibroblasts, a major player in fibrosis [16]. Thus, OPN may act as both a regulatory and a structural molecule in tissue fibrosis.

There is a vast literature detailing OPN's potential roles and location of its expression in both renal development and disease $[17,18]$. Several studies reported an association of upregulation of glomerular OPN expression with macrophage accumulation and progressive renal injury in an anti-GBM GN model [19]. Anti-OPN antibody treatment reduced proteinuria and prevented loss of renal function, suggesting OPN to be a proinflammatory molecule [20]. However, one study showed a lack of in vivo function of OPN in an anti-GBM GN model using OPN null mice [21]. A potential role of OPN in renal interstitial fibrosis has been reported [22]. OPN may modulate angiotensin-II-induced inflammation, oxidative stress, and fibrosis of the kidney [23]. Recently, a critical role of OPN in diabetic nephropathy has been reported $[24,25]$.

In our rat model for anti-GBM GN, disease is induced by immunization with a T-cell epitope $\mathrm{pCol}(28-40)$ derived from $\alpha 3$ chain of type IV collagen [26, 27]. The disease undergoes two distinct overlapping stages: $\mathrm{CD} 4^{+}$ T-cell-mediated glomerular inflammation followed by severe glomerular fibrosis. In the present study, we investigated whether OPN expression was associated with glomerular fibrosis in this model.

\section{Methods}

Antigen Preparation and GN Induction

Nephritogenic T-cell peptide pCol(28-40) was commercially synthesized and dissolved in milli-Q water at a 1-mM concentration. Female Wistar-Kyoto (WKY) rats (4-6 weeks) were purchased from Harlan (Indianapolis, Ind., USA). Rats were immunized with $\mathrm{pCol}(28-40)(0.125 \mu \mathrm{mol})$ emulsified in complete
Freund adjuvant (CFA), in one hind footpad and at the base of the tail. Rats immunized with CFA alone served as controls. All animal procedures were approved by IACUC.

Evaluation of GN Severity and Glomerular Fibrosis

Kidney tissues were fixed in Bouin's solution, and glomerular injury score was calculated using HE-stained sections [27]. The tissue sections were also stained by PAS to reveal fibrotic tissue. Three sections were randomly selected from each animal. All glomeruli on the sections were counted and classified into normal, inflammatory and fibrotic. Glomeruli with fibrotic crescent lesions were counted and expressed as a percentage of all glomeruli. A portion of kidney was snap-frozen or fixed in $4 \%$ paraformaldehyde (PFA) for immunofluorescence. Immunofluorescence was performed on the kidney section from immunized rats with antibodies to collagen $1 \alpha 1$, collagen $4 \alpha 1$ or $4 \alpha 2$ to discriminate glomerular sclerosis from fibrosis.

Isolation and Analysis of Glomerular Cells

After the kidneys had been perfused in situ with PBS, glomeruli were isolated to a purity of $>95 \%$. The isolated glomeruli were digested with a mixture of collagenase IV and Accutase ${ }^{\text {TM }}$ (eBioscience, San Diego, Calif., USA) at room temperature for 30 min with gentle stirring. The cells were released by repeated pipetting. Dead cells were further removed by Ficoll density centrifugation. For immunofluorescence, the cells were placed in each well of 4-well chamber slide overnight (Fisher, Rochester, N.Y., USA). Intracellular staining for OPN was performed using a kit of Golgi-stop reagent (Cytofix/Cytoperm; BD, San Diego, Calif., USA) following the manufacturer's instructions. OPN was stained with biotin-labeled mouse monoclonal antibody to OPN (Akm2A1; Santa Cruz Biotech, Santa Cruz, Calif., USA) followed by Texas Red-labeled avidin. The cells were further incubated with FITC-labeled monoclonal antibodies to CD4, CD $8 \alpha$, CD11b/c, RT.1B, CD90, and $\alpha$-smooth muscle actin ( $\alpha$ SMA).

\section{Detection of Glomerular OPN Expression}

DNA microarray and RT-PCR were used to detect OPN mRNA. Briefly, total RNA isolated from purified glomeruli (Ambion, Austin, Tex., USA) was probed with oligo DNA GEArray ${ }^{\circledR}$ which contained 120 genes for ECM and adhesion molecule genes (SA Biosciences, Frederick, Md., USA) for expression level of those genes following the manufacturer's instructions. For conventional RT-PCR, cDNA was synthesized (Sigma, St. Louis, Mo., USA), and PCR was carried out using a pair of primers specific for rat OPN (forward primer 5'-GCCCTGAGCTTAGTTCGTTG-3' and reverse primer 5'-TCCTGTAAGTTTGCCTGCCT-3') for 35 cycles. Real-time PCR was performed using QuantiTect SYBR Green PCR Kits (Qiagen, Valencia, Calif., USA) on iCycler (BioRad, Hercules, Calif., USA). Genes acidic ribosomal phosphoprotein P0 and GAPDH were used as controls.

Immunofluorescence and Western blot were utilized to detect OPN protein. For immunofluorescence, frozen kidney sections were first incubated with biotin-labeled antibody Akm2A1 (1:200) or control IgG followed by Texas Red-labeled avidin. The sections were further stained with FITC-labeled anti-rat RT.1B monoclonal antibody or SR-13 monoclonal antibody, which reacts with both GBM and tubular basement membrane, and subsequently counterstained by DAPI [27]. Three-color images were recorded by a digital fluorescent microscope (H550L; Nikon, Ja- 
Fig. 1. Glomerular fibrosis in WKY rats after immunization with nephritogenic T-cell epitope pCol(28-40) derived from collagen IV $\alpha-3$ chain. a Time course of glomerular fibrosis. Glomerular fibrosis is expressed as a percentage of glomeruli with fibrotic crescent lesions. This graph is based on 58 rats. Each open circle represents one individual. The bar beneath the graph indicates general disease stages. b Number of deaths among immunized rats post-immunization. Autopsy demonstrated severe fibrosis in all rats. c Fibrosis in glomeruli demonstrated by PAS staining. d, e Immunofluorescences on collagen $1 \alpha 1$ (red, d), or collagen $4 \alpha 2$ (red, e); arrowheads outline fibrotic tissue in $\mathbf{e}$; the sections were counterstained for collagen $4 \alpha 3$ (green) to show damaged GBM. $\times 400$. (Colors refer to online version only.)

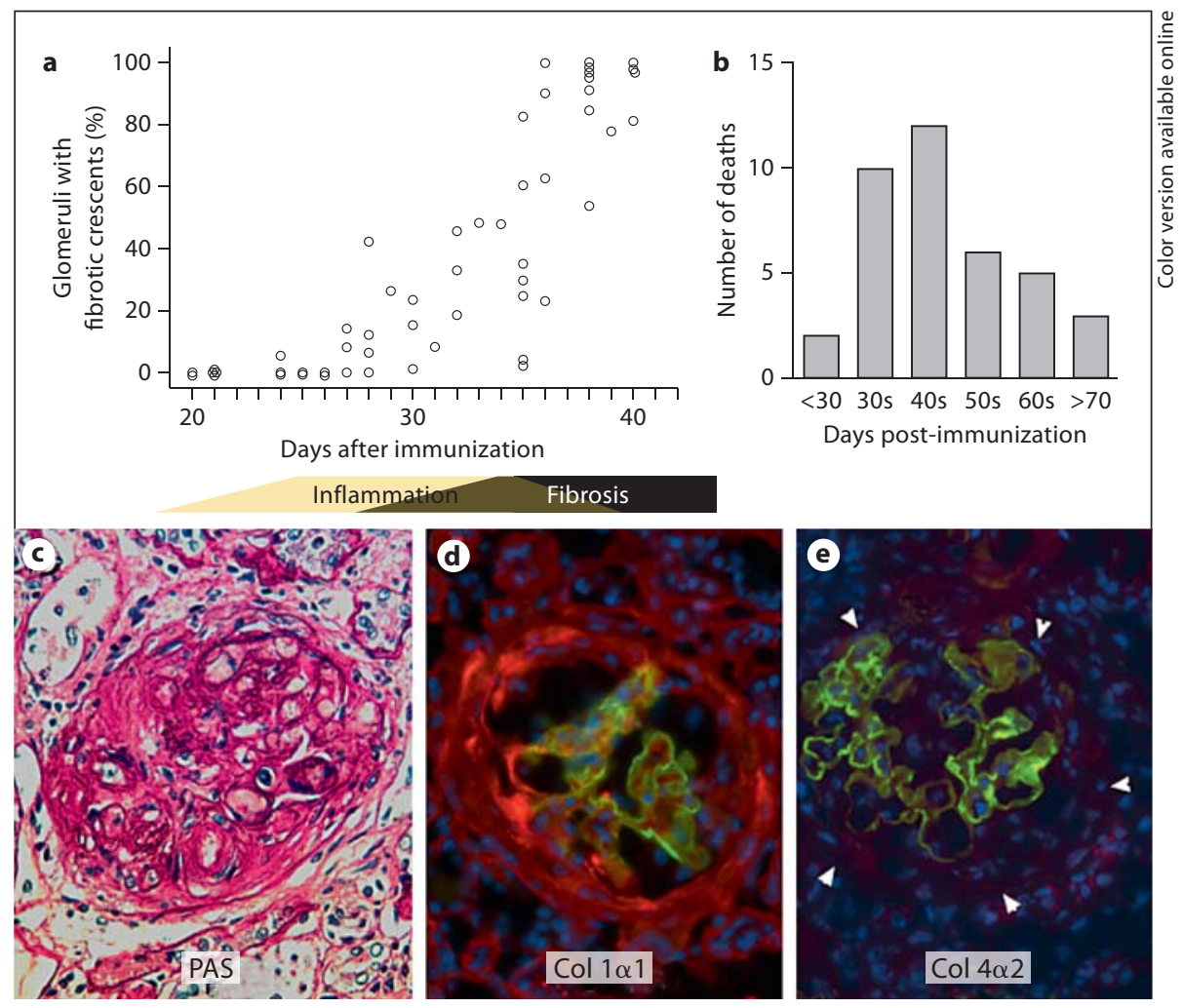

pan). To extract glomerular ECM, purified glomeruli $\left(2 \times 10^{4}\right)$ were subjected to repeated sonications followed by intensive washing. The ECM pellet was resuspended in water and lyophilized. The ECM proteins were separated either by SDS-PAGE or two-dimensional (2-D) electrophoresis. For Western blot, the membranes were stained with biotin-labeled antibody Akm2A1. An enhanced chemiluminescence kit (Sigma) was applied to reveal OPN proteins.

\section{Detection of Cellular Senescence}

Cultured cells or kidney sections were fixed with $0.25 \%$ of glutaraldehyde for $10 \mathrm{~min}$ and washed with cold PBS. A special staining solution from a kit was added to the cells or tissue sections and incubated at $37^{\circ} \mathrm{C}$ overnight (senescence-associated $\beta$-galactosidase (SA- $\beta$-gal) Cellular Senescence Assay Kit; Cell Biolabs, San Diego, Calif., USA). First, renal pathology, i.e. normal inflammatory and fibrotic, was determined on an HE-stained section for each rat. Three rats were selected for each stage. 15 glomeruli from three sections for each individual were randomly selected and digitally recorded. SA- $\beta$-gal ${ }^{+}$foci were counted on the digital images. Average SA- $\beta$-gal ${ }^{+}$foci/glomerulus were calculated for each individual. In some cases, the sections were further stained for $\alpha$ SMA and nuclear counterstaining by propidium iodide for colocalization. Fluorescent and bright-field images were taken at the same location, and synthesized to reveal location of $\alpha \mathrm{SMA}$, nuclei and SA- $\beta$-gal activity.

\section{Results}

\section{Time Course of Glomerular Fibrosis in}

pCol(28-40)-Induced Anti-GBM GN

Glomerular inflammation plateaued at days 28-35 after pCol(28-40) immunization. Glomerular fibrosis was histologically observed as early as days 25-30 (fig. 1a). After a robust increase in the number of fibrotic glomeruli between days 35 and 40, fibrotic crescents were observed in $80-90 \%$ of glomeruli after day 40 (fig. 1a). Glomerular fibrosis led to development of ascites and high mortality (fig. 1b). Fibrotic crescents were revealed by PAS staining and immunofluorescence on collagen $1 \alpha 1$ deposition (fig. 1c, d). On the other hand, collagen $4 \alpha 1$ or $4 \alpha 2$ deposition was not found (fig. 1e). Fibrotic crescents occupied a very limited space in Bowman's capsule at early stages, coexisting with numerous infiltrating leukocytes, and then gradually expanded through the entire glomerulus by days 45-50. After onset of fibrosis, infiltrating leukocytes gradually vanished from the glomeruli. In many cases, GBMs, or its fragments, were no longer present after day 50, suggesting a complete destruction of GBM and capillary tufts. 


\section{Upregulation of Glomerular OPN Expression after}

\section{Glomerular Fibrosis}

GN stage (normal, early and peak inflammation, and fibrosis) in each immunized rat was histologically determined. According to their disease stage, total glomerular RNAs from the same rats were selected for experiments. ECM gene expression patterns were compared among the normal glomeruli (day 0), those from early (day 20), peak inflammation (day 30), and fibrosis stage (day 40) (2 samples/stage). Expression of at least 6 genes, including OPN, secreted protein acidic and rich in cysteine (SPARC), was significantly upregulated in the fibrotic glomeruli as compared to normal or inflamed glomeruli (fig. 2a). Although RNA for SPARC was most abundant at the fibrotic stage, upregulation of its expression occurred during the inflammatory stage. This study solely focused on OPN. Glomerular OPN expression was further examined by RT-PCR (3 rats/stage). Unlike DNA microarray, RT-PCR detected OPN expression in all three stages (fig. 2b). However, the expression levels were significantly different between the stages. Fibrotic glomeruli showed much higher levels of OPN expression than the others. Quantitative PCR showed that fibrotic glomeruli had an approximately 16- and 10-fold more OPN mRNA than normal and inflamed glomeruli, respectively (fig. 2c).

OPN expression was examined at the protein level. Glomeruli were isolated at day 25 (inflammatory group) and day 45 (fibrotic group) from 3 rats/group. Glomeruli isolated from 3 CFA-immunized rats at day 45 were used as a control (CFA group). ECM proteins were extracted from the same numbers of glomeruli $\left(2 \times 10^{4}\right)$. Western blot showed the presence of OPN proteins in all three groups as multiple major bands, ranging from 55 to 60 $\mathrm{kDa}$, with a minor band at $66 \mathrm{kDa}$ (fig. 2d). It is well known that OPN is expressed as multiple isoforms ranging from 44 to $66 \mathrm{kDa}$ in various tissues [18]. Thus, the majority of detected OPN may represent an isoform, which may differ from that in the normal tubules. Three groups showed significant difference in OPN quantities: fibrotic glomeruli (day 45) showed a much larger quantity of OPN, with several bands of low molecular weights, than the other two groups (inflammatory and CFA). Densitometry showed that OPN from day 45 was 15 - and 11-fold higher than that of the CFA and inflammatory group, respectively.

Due to variations in phosphorylation, as well as matrix metalloproteinase-mediated cleavages, OPN may be present as multiple isoforms. Glomerular ECM proteins were analyzed by 2-D Western blot. Three major forms of

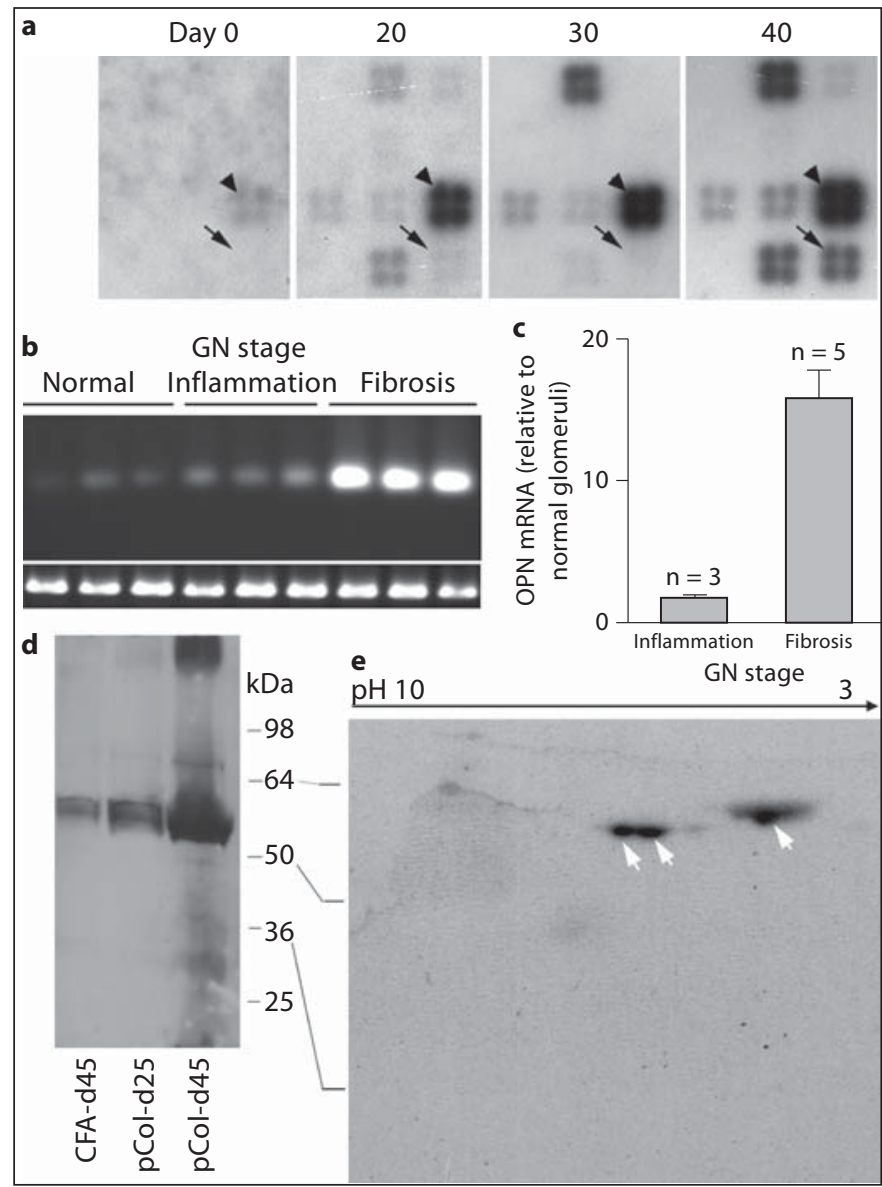

Fig. 2. Upregulation of glomerular OPN expression during fibrotic stage in the immunized WKY rats. a Detection of OPN mRNA (arrows) at different time points post-immunization with DNA microarray for ECM-related genes. Only a portion of the membrane is shown. SPARC is indicated by arrowheads. b RT-PCR detection of OPN mRNA. GN stages are indicated and three representative samples are shown for each stage. Expression of a control gene acidic ribosomal phosphoprotein (P0) is shown at the bottom. c Quantitative RT-PCR detection of OPN mRNA at different stages. Levels of OPN mRNA are expressed as multiples of that of normal glomeruli. d Western blot detection of OPN protein in glomeruli isolated from different groups at days 25 and 45. $p C o l, p C o l(28-40)$-immunized rat. Only one representative sample from each group is shown. e 2-D Western blot characterization of OPN protein isolated from glomeruli with severe fibrosis. Arrows indicate three isoforms of OPN.

OPN, which migrated to a very low $\mathrm{pH}$ area with molecular weights of $55-56 \mathrm{kDa}$, were detected in the fibrotic group (day 45) (fig. 2e). However, 2-D Western blot failed to detect OPN in the other two groups, or low molecular weight OPNs on day 45 , presumably due to a limited amount of OPN (data not shown). 


\section{Massive Deposition of OPN on Glomerular}

\section{Crescent Lesions}

Three rats were used for each stage. Normal rats showed a small quantity of extracellular OPN localized to Bowman's capsule (fig. 3a). Observation on serial sections showed that these short linear OPN deposits had a polarized distribution. On many occasions, OPN proteins were co-localized with RT.1B-expressing cells, which aggregated to one pole (fig. 3b). Intracellular OPN in granular form was detected in some tubular epithelial cells of normal rats as previously reported (fig. 3a, b).

We next investigated the OPN distribution pattern in the glomeruli after disease induction. Inflammatory foci, i.e. RT. $1 \mathrm{~B}^{+}$areas, showed a polarized distribution in the inflamed glomeruli (day 30) (fig. 3c, d). Clustered granular OPN proteins were present in Bowman's capsule (fig. 3c); those granules were not closely associated with RT.1B ${ }^{+}$areas (fig. 3d). At this stage, a few RT.1B ${ }^{+}$cells had intracellular OPN. In contrast, at the fibrotic stage, a much larger quantity of OPN was observed (fig. 3e-h). At the early fibrotic stages (day 35), fibrotic tissue co-exited with RT.1B ${ }^{+}$ infiltrating leukocytes (fig. 3f). OPN proteins assembled in bold linear deposits, mainly localized to the origin of the fibrotic lesion in Bowman's capsule (fig. 3e, f). OPN deposition and RT. $1 \mathrm{~B}^{+}$were adjacent to each other but not overlapping. As glomerular fibrosis progressed, a circular deposition of OPN was frequently observed (fig. 3g, h). The OPN deposition, which resembled bundles of parallel fibers, co-localized to the fibrotic crescents (fig. 3g). At this stage, RT. $1 \mathrm{~B}^{+}$inflammatory loci had greatly contracted (fig. 3h). Thus, the majority of OPN during fibrosis was present as an ECM protein deposited in fibrotic tissues.

Rats with severe glomerular fibrosis could be recognized by the presence of severe anasarca. Glomerular OPN in these rats was detected as extremely dense deposits localized to the fibrotic crescent (fig. 3i). In the rats with end-stage disease, circular deposition of OPN was found on the circular fibrotic crescent, which occupied most of a glomerulus, with few, if any, GBM fragments left (fig. 3j). Due to massive deposition of collagen fibers, trapped IgG might lead to a false positive result for OPN. We carefully examined specificity of the staining. Both normal rabbit IgG and mouse control IgG isotype failed to stain the fibrotic areas (fig. 4).

Multinucleated $\alpha S M A^{+} C D 90^{-} R T .1 B^{-} C D 11 b / c^{-}$Cells Were the Major Source of OPN during Glomerular Fibrosis

We now wished to determine which type of cells produced OPN during glomerular fibrosis. Glomerular cells were then isolated at day 40. Two-color immunofluores-
SR-13 (g) vs. OPN (r)
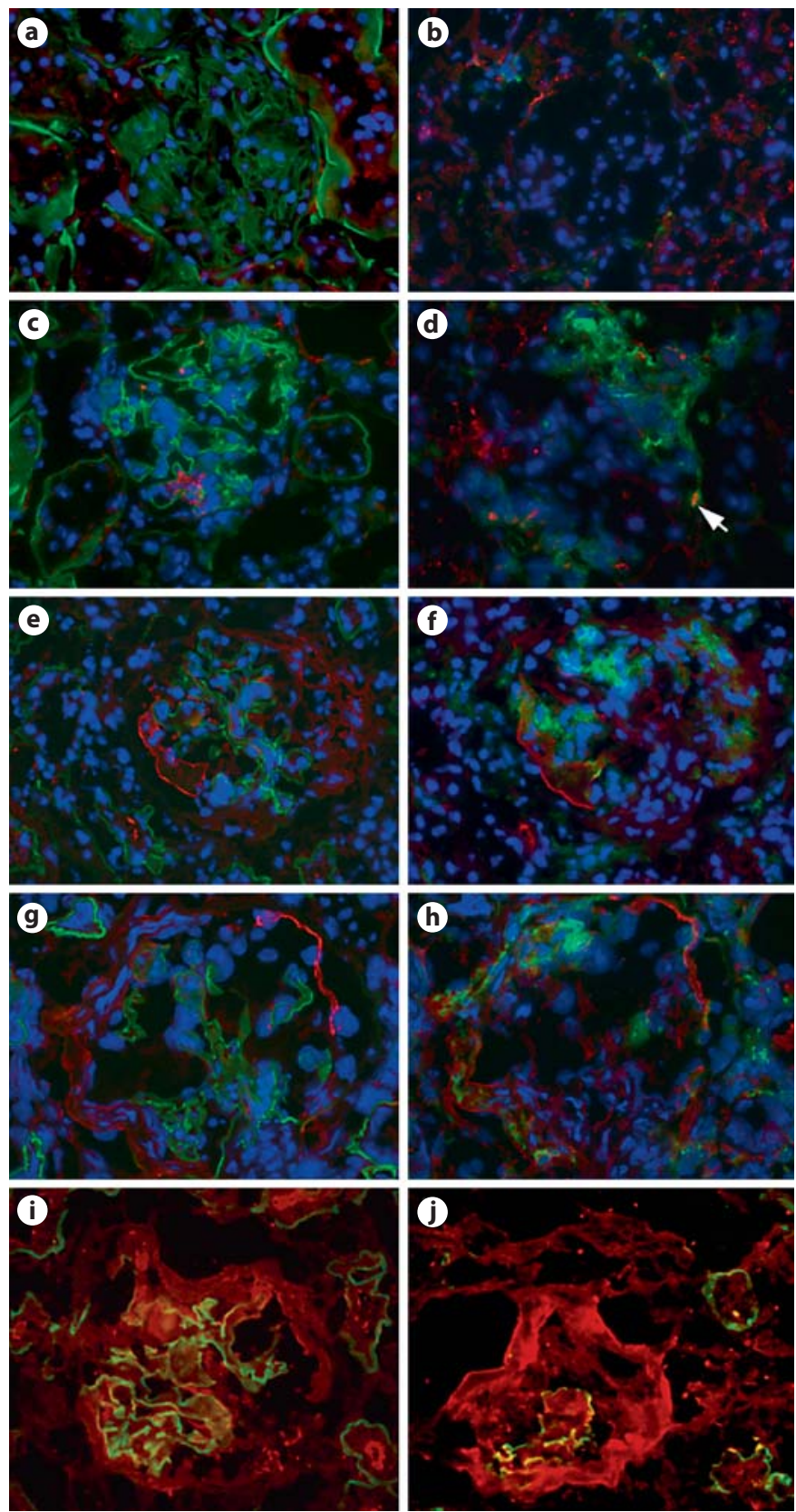

Fig. 3. Immunofluorescent localization of OPN proteins in normal glomeruli or in glomeruli at different stages after immunization with $\mathrm{pCol}(28-40)$. $\mathbf{a}-\mathbf{h}$ Pairs of two adjacent sections were stained for localization of OPN (red) in glomeruli at different stages (see below). GBM or RT.1B was stained green to reveal glomerular structure or inflammatory foci. Sections were counterstained by DAPI to reveal nuclei. a, b Normal glomeruli. c, $\mathbf{d}$ Early inflammation. Arrow in $\mathbf{d}$ shows a RT. $1 \mathrm{~B}^{+} \mathrm{OPN}^{+}$cell. e, $\mathbf{f}$ Late inflammation with early fibrosis. $\mathbf{g}, \mathbf{h}$ Mid fibrosis. i OPN (red) deposition on fibrotic tissue in a glomerulus at mid-late fibrosis. Note the thickened GBM (green). $\mathbf{j}$ OPN (red) deposition on a circular crescent at late fibrosis. Note only a small fragment of GBM (green) remained. $\times 400$. (Colors refer to online version only.) 

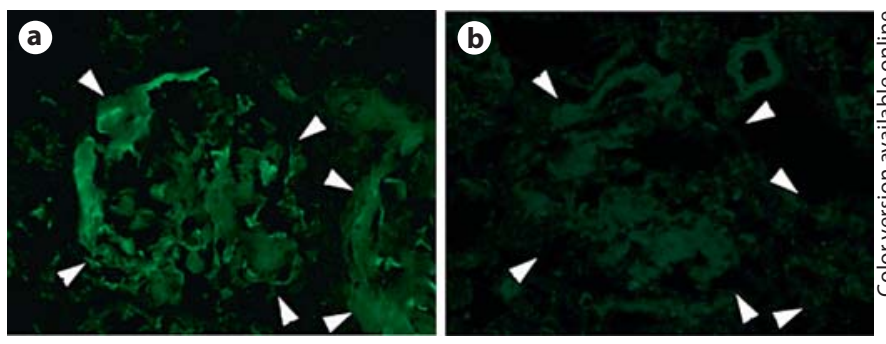

Fig. 4. Verification of specific binding of anti-OPN antibody to OPN. a Kidney section shows two fibrotic glomeruli after being stained with anti-OPN antibody. Note bold depositions of OPN on fibrotic tissue. b An adjacent section after incubation with IgG control. Note no special staining even after overexposure. Glomeruli are outlined by arrowheads. $\times 400$.

cence was performed to determine which cells expressed OPN after Golgi protein secretory pathway was blocked in vitro. Intracellular OPN protein was not detected in any $\mathrm{CD}^{+} \mathrm{T}$ cells or $\mathrm{CD} 8 \alpha^{+}$cells (fig. $5 \mathrm{a}, \mathrm{d}$ ). Less than $2 \%$ of $\mathrm{CD} 11 \mathrm{~b} / \mathrm{c}^{+}$cells (macrophages and dendritic cells) showed cytoplasmic OPN (fig. 5b). Intracellular OPN was detected in approximately $5.4 \%$ of RT.1B ${ }^{+}$cells (fig. $5 \mathrm{c}$ ). We next determined OPN expression in CD90 (Thy-1) ${ }^{+}$ cells. Since mesangial cells also expressed CD90, the nonmesangial cells were identified by their fibroblast like morphology. Despite the presence of numerous normal $\mathrm{CD}^{2} 0^{+}$fibroblast-like cells, OPN was detected in only $<1 \%$ of them (fig. 5e). Approximately $5 \%$ of $\mathrm{CD}^{+} 0^{+}$cells were found to contain 3-4 nuclei. Intracellular OPN was detected in approximately $30 \%$ of those cells (fig. 5f). A population of $\mathrm{CD}^{-} 0^{-}$giant cells (approximately $1 / 5$ of $\mathrm{CD} 90^{+}$fibroblasts) each containing as many as 12 large nuclei were observed (fig. 5g). Various levels of OPN expression were found in this subset of cells, with distribution of high level $39.1 \%$, middle to low level $11.6 \%$, and none $49.3 \%$ (fig. $5 \mathrm{~g}, \mathrm{~h}$ ). Multinucleated giant cells of macrophage origin have been reported in glomeruli in another anti-GBM GN model [26]. However, the $\mathrm{CD}^{-} 0^{-} \mathrm{OPN}^{+}$giant cells in our model did not express RT.1B, CD11b/c and CD68 (ED-1) (data not shown), which are known markers for macrophage-derived giant cells [28]. On the other hand, the $\mathrm{CD}^{-} 0^{-} \mathrm{OPN}^{+}$multinuclear cells contained $\alpha \mathrm{SMA}$ and morphologically resembled myofibroblasts (fig. 5h, i). Thus, it is likely that these giant cells probably originated from $\mathrm{CD} 90^{+}$myofibroblasts. Because glomerular cells were used for staining only $12 \mathrm{~h}$ after isolation, this subset of cells was not due to prolonged in vitro culture. The experiments were repeated in 5 immunized rats with similar results.

Glomerular Senescent Myofibroblasts Express OPN

\section{In vivo Identification of OPN Producing} Multinucleated Myofibroblasts

$\mathrm{OPN}^{+}$multinucleated cells contained $\alpha \mathrm{SMA}$. Thus, $\alpha$ SMA was used as a marker to locate multinucleated cells in vivo. Immunofluorescence showed a very faint linear staining for $\alpha$ SMA surrounding Bowman's capsule in normal kidney. In contrast, an $\alpha \mathrm{SMA}^{+}$cellular layer was observed to surround fibrotic tissue in fibrotic glomeruli (fig. 6a, d). In some cases, a few clustered $\alpha \mathrm{SMA}^{+}$cells were adjacent to the inner edge of the fibrotic tissue (fig. 6b). The $\alpha \mathrm{SMA}^{+}$cells, especially those surrounding the fibrotic tissue, often contained multinuclei (fig. 6a, b). As a control, $\alpha \mathrm{SMA}^{+}$smooth muscle cells of arteries near fibrotic glomeruli contained a single nucleus (fig. 6b). Two-color immunofluorescence on the section of fibrotic kidneys revealed that OPN deposition was surrounded by an $\alpha \mathrm{SMA}^{+}$cell layer with many of these cells containing OPN (fig. 6d). High magnification further showed intracellular OPN in $\alpha \mathrm{SMA}^{+}$ cells, some of which contained more than one nucleus (fig. 6e). Thus, both in vitro and in vivo experiments demonstrated that $\alpha \mathrm{SMA}^{+}$myofibroblasts were the major source of OPN.

\section{Multinucleated Myofibroblasts Are Senescent Cells}

We next investigated which mechanisms led to multinucleation in those myofibroblasts. Cellular senescence is one of the mechanisms for multinucleation. Expression of SA- $\beta$-gal has been widely used for detection of cellular senescence. $\mathrm{CD} 90^{+}$myofibroblasts from late inflammatory/early fibrotic stage showed a lack of SA- $\beta$ gal activities (fig. 7a). However, SA- $\beta$-gal activity was detected in the multinucleated $\alpha \mathrm{SMA}^{+}$cells (fig. $7 \mathrm{~b}$ ). Thus, some of multinucleated $\mathrm{OPN}^{+} \alpha \mathrm{SMA}^{+} \mathrm{CD} 90^{-}$cells were probably senescent myofibroblasts. We next examined whether SA- $\beta$-gal ${ }^{+}$cells were present in vivo. Kidney sections from different stages were used (three for each stage). SA$\beta$-gal ${ }^{+}$foci were not detected in normal glomeruli, although strong $\beta$-galactosidase activities were observed in normal tubular epithelial cells as previously reported (fig. 8a). Single weak SA- $\beta$-gal ${ }^{+}$focus was occasionally detected in glomeruli at late inflammatory/early fibrotic stage (16.2\%) (fig. $8 b)$. On the other hand, SA- $\beta$-gal ${ }^{+}$cells were observed in a high frequency (86.3\%) in glomeruli with fibrotic crescents (fig. $8 \mathrm{c}$, d). These SA- $\beta$-gal ${ }^{+}$cells, which are mostly embedded in or surrounded by fibrotic tissue of affected glomeruli, were co-localized to the $\alpha \mathrm{SMA}^{+}$area (fig. 8e). There were up to six SA- $\beta$-gal ${ }^{+}$foci in a single fibrotic glomerulus (fig. 8f). Although counting SA- $\beta$-gal ${ }^{+}$foci on a 2 -D image may not be sufficient- 

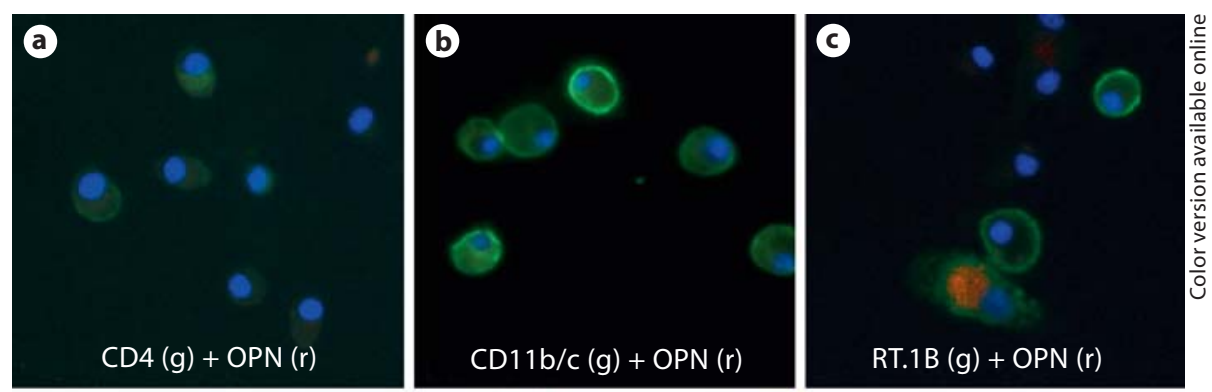

Fig. 5. Immunofluorescent identification of OPN-expressing glomerular cells in vitro. Glomerular cells were isolated from a rat at day 40 post-immunization with pCol(28-40). a-f Various cells were doubly stained for cell markers (green, as indicated) and OPN (red) after incubation with Golgi block reagent. Nuclei are revealed by DAPI (purple). g, h Giant cells with multinuclei were co-stained for OPN (red) with CD90 (green in $\mathbf{g}$ ) or $\alpha$ SMA (green in $\mathbf{h}$ ). i Multinucleated giant cells, which were not treated by Golgi block reagent, were stained for $\alpha$ SMA (green). Inset: PCR detection of expression of OPN isolated from adhesive multinucleated cells. The left lane shows DNA markers for 300, 400, and 500 bps, respectively. $r=$ Red; $g=$ green. $\times 600$. (Colors refer to online version only.)
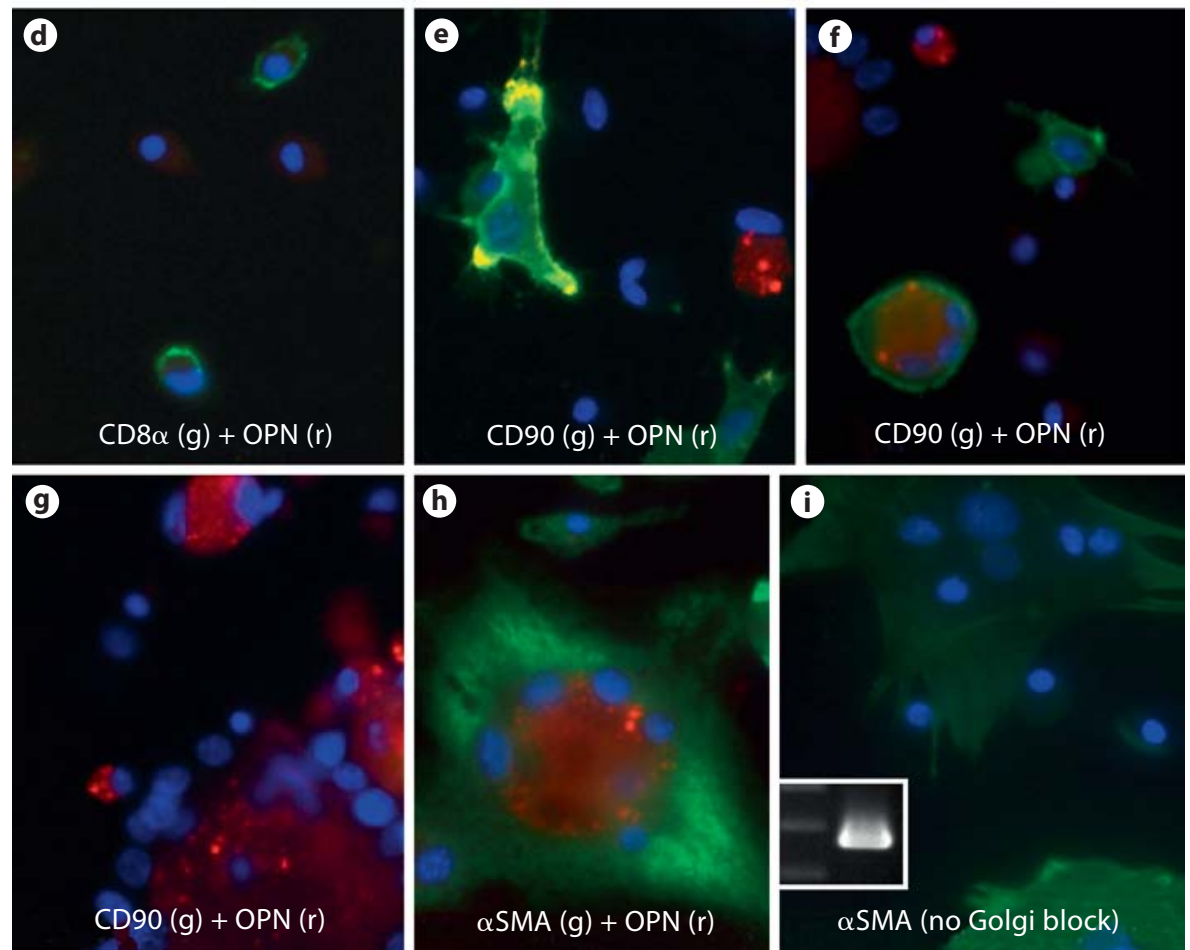

ly accurate [29], their presence in fibrotic tissue, but not in normal or inflammatory glomeruli, was clearly demonstrated.

\section{Discussion}

Recently, a critical role of OPN in fibrosis in several organs has been reported [12-15]. Several studies have speculated that OPN may also play a role in glomerular fibrosis $[20,22,23]$. The present study revealed a strong association between upregulation of OPN and progression of glomerular fibrosis. Our time course studies showed, at both the mRNA and protein level, that the expression of OPN was greatly upregulated only after fibrosis. Furthermore, we identified novel senescent myofibroblasts to be a major source of massive OPN during fibrosis.
Upregulation of OPN has been reported in several other renal disease models $[19,20,30,31]$. Similar to our findings, one study reported OPN mRNA in glomeruli [27]. Using in vitro immunofluorescence for detection of OPN protein after Golgi block, we were able to identify novel multinucleated cells to be the major source of glomerular OPN. Although they lacked CD90 expression, these $\mathrm{OPN}^{+}$ giant cells expressed $\alpha$ SMA but not markers such as RT.1B, $\mathrm{CD} 1 \mathrm{~b} / \mathrm{c}, \mathrm{ED}-1 \mathrm{c}$ for macrophage-origin giant cells. In addition, they were morphologically similar to $\alpha \mathrm{SMA}^{+} \mathrm{CD} 90^{+}$ myofibroblasts. A previous study showed that the loss of CD90 expression in fibroblasts leads to more severe lung fibrosis [32]. Thus, these cells were probably a subset of myofibroblasts. Cellular senescence has been reported in prolonged wound-related inflammation by affecting wound closure [33]. Our experiments demonstrated the presence of SA- $\beta$-gal activities in these cells both in vi- 
Fig. 6. Immunofluorescent detection of $\alpha \mathrm{SMA}^{+}$multinucleated cells in fibrotic glomeruli. a $\alpha \mathrm{SMA}^{+}$myofibroblasts surrounding fibrotic tissue contained multinuclei. b A cluster of $\alpha \mathrm{SMA}^{+}$myofibroblasts with multi-nuclei (arrows) adjacent to the inner edge of fibrotic tissue. c Single nucleated $\alpha \mathrm{SMA}^{+}$cells in two arteries (asterisks). Arrow indicates a double-nucleated $\alpha \mathrm{SMA}^{+}$cell in fibrotic glomerulus. Right three panels of $\mathbf{a}-\mathbf{c}$ are enlarged images of the rectangular area for DAPI, $\alpha S M A$, or synthesized one, respectively. d $\alpha \mathrm{SMA}^{+}$cell layer (green) surrounding OPN deposition (red) at low magnification. $\times 100$. Note that cytoplasm of several $\alpha \mathrm{SMA}^{+}$cells are $\mathrm{OPN}^{+}$(arrowhead). e High magnification shows $\alpha \mathrm{SMA}^{+} \mathrm{OPN}^{+}$ cells (arrows) in the $\alpha \mathrm{SMA}^{+}$cellular layer surrounding OPN deposition. Sections for a-c were from PFA-fixed tissue. Sections for $\mathbf{d}$ and e were from snap-frozen tissue. $\times 300$; enlarged images $\times 600$. (Colors refer to online version only.)

Fig. 7. Cultured multinucleated myofibroblasts express SA- $\beta$-gal. a Mononuclear myofibroblasts isolated from late inflammatory/early fibrotic stage show a lack of $\beta$-gal activity. b Multinucleated myofibroblasts isolated from late fibrotic stage display strong $\beta$-gal activity (blue). (Colors refer to online version only.)

Glomerular Senescent Myofibroblasts Express OPN
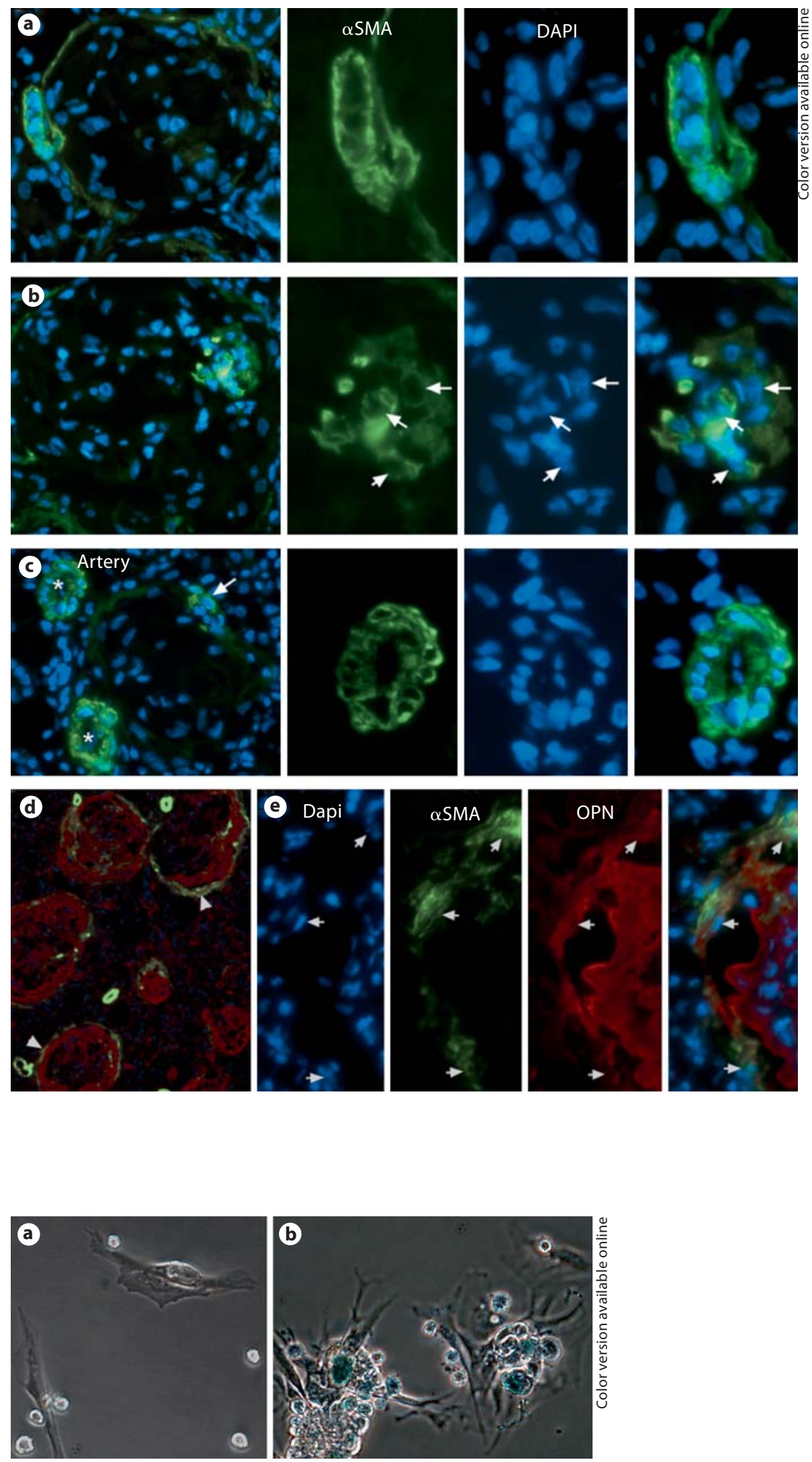

Am J Nephrol 2010;32:262-271 

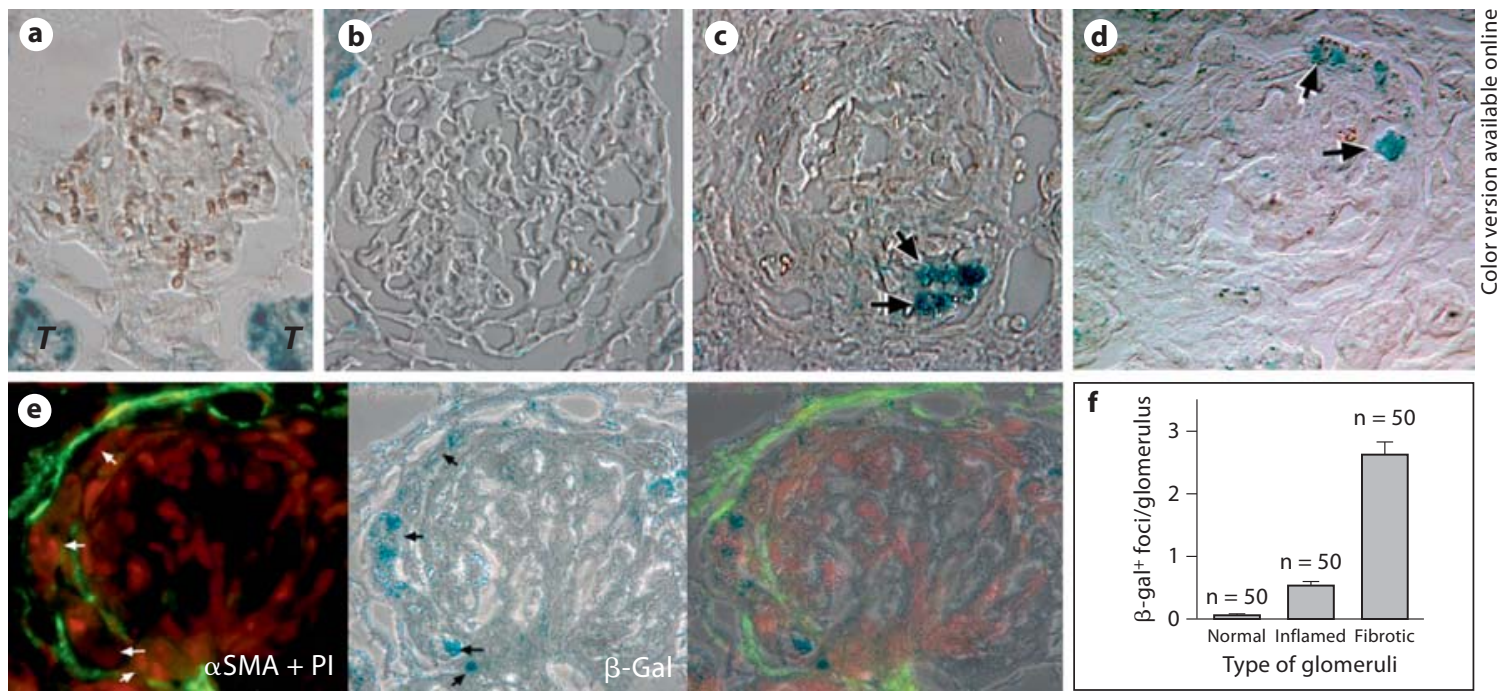

Fig. 8. Presence of SA- $\beta$-gal activities in the fibrotic glomeruli. a Normal glomerulus showing a lack of enzyme activity. Note enzyme activities in tubules, which are indicated by T. b Absence of enzyme activity in an enlarged glomerulus with inflammation. c, $\mathbf{d}$ Representative micrographs show multiple foci with strong $\beta$-gal activates (arrows) in fibrotic glomeruli. e Co-localization of $\beta$-gal ${ }^{+}$foci (arrows) with $\alpha$ SMA (green). The section was counterstained with propidium iodide to show nuclei (red). f Summary of $\beta$-gal ${ }^{+}$foci per glomerulus at three stages. (Colors refer to online version only.)

tro and in vivo. It is likely that these multinucleated $\mathrm{OPN}^{+} \alpha \mathrm{SMA}^{+} \mathrm{CD} 90^{-}$cells were terminally differentiated myofibroblasts with loss of CD90 expression. It would be interesting to determine the relationship between upregulation of OPN, cellular senescence, and progression of glomerular fibrosis. This may be related to another interesting question, i.e. why only a fraction of the multinucleated myofibroblasts expressed OPN? It remains unclear. One possibility is that the multinucleated myofibroblasts are a group of cells with differentiation stages. Alternatively, OPN expression in these cells is transient.

Which role may OPN play in glomerular fibrosis? Different phosphorylation patterns in OPN or its various isoforms are related to its special functions as a regulatory molecule [18]. It is not surprising that OPNs isolated from various tissues such as bone tissue and normal kidneys have a molecular weight of 44 and $66 \mathrm{kDa}$, respectively. In our study, the majority of OPN was detected as a protein with a molecular weight of $56 \mathrm{kDa}$, suggesting a different isoform from those normally expressed in the tubules. Thus, determination of the structures of three OPN isoforms found in our model will be critical for our understanding of its role as a regulatory or structural molecule. As a regulatory molecule, OPN may be required for differentiation of myofibroblasts, a major play- er in fibrosis $[16,18]$. As a structural protein, OPN may facilitate assembly of ECM protein to form fibrotic tissue. Disorganization of matrix in skin incisions in OPN mutant mice suggests involvement of OPN in ECM protein assembly [15]. The above hypothesis is currently being tested using neutralizing antibody to OPN.

Expression of another ECM protein SPARC was also upregulated during both inflammatory and fibrotic stages in our model. In fact, SPARC was the most abundant ECM molecule during fibrosis. SPARC has been known to be involved in fibrosis/scar formation. However, it is less clear whether SPARC plays a role in glomerular fibrosis. It would be interesting to test whether blocking both SPARC and OPN leads to an additive inhibitory effect on fibrosis in GN.

\section{Acknowledgments}

This work is supported by the NIH grant R01 DK60029 (Y.H.L.) and R01 DK77857 (Y.H.L.). J.M. was supported by O'Brian Inflammation Center, Renal Division, Baylor College of Medicine. We thank Drs. W. Butler, University of Texas Houston, and Y. Sado, Okayama University, Japan, for providing antibodies to rat OPN and glomerular basement membrane, and Ms. K. Parker for preparing the manuscript. 


\section{References}

$\checkmark 1$ Chatziantoniou C, Boffa JJ, Tharaux PL, Flamant M, Ronco P, Dussaule JC: Progression and regression in renal vascular and glomerular fibrosis. Int J Exp Pathol 2004;85:1-11.

$\checkmark 2$ Liu Y: Renal fibrosis: new insights into the pathogenesis and therapeutics. Kidney Int 2006;69:213-217.

$\checkmark 3$ Eddy AA: Molecular basis of renal fibrosis. Pediatr Nephrol 2000;15:290-301.

4 Boffa JJ, Lu Y, Placier S, Stefanski A, Dussaule JC, Chatziantoniou C: Regression of renal vascular and glomerular fibrosis: role of angiotensin II receptor antagonism and matrix metalloproteinases. J Am Soc Nephrol 2003; 14:1132-1144.

5 François H, Placier S, Flamant M, Tharaux PL, Chansel D, Dussaule JC, Chatziantoniou $\mathrm{C}$ : Prevention of renal vascular and glomerular fibrosis by epidermal growth factor receptor inhibition. FASEB J 2004; 18:926-928.

-6 Franzén A, Heinegård D. Isolation and characterization of two sialoproteins present only in bone calcified matrix. Biochem J 1985;232:715-724

$\checkmark 7$ Denhardt DT, Noda M, O’Regan AW, Pavlin D, Berman JS: Osteopontin as a means to cope with environmental insults: regulation of inflammation, tissue remodeling, and cell survival. J Clin Invest 2001;107:1055-1061.

$>8$ O’Regan AW, Nau GJ, Chupp GL, Berman JS: Osteopontin (Eta-1) in cell-mediated immunity: teaching an old dog new tricks. Immunol Today 2000;21:475-478.

-9 Giachelli CM, Scatena M, Wada T: Osteopontin: Potential roles in vascular function and dystrophic calcification. J Bone Miner Metab 1997;15:179-183.

$\checkmark 10$ Bird L: Asthma and allergy: opposing roles for osteopontin. Nature Rev Immunol 2007; $7: 417$.

- 11 Chabas D, Baranzini SE, Mitchell M, Bernard CCA, Rittling SR, Denhardt DT, Sobel RA, Lock C, Karpuj M, Pedotti R, Heller R, Oksenberg JR, Steinman L: The influence of the proinflammatory cytokine, osteopontin, on autoimmune demyelinating disease. Science 2001;294:1731-1735.

-12 Pardo A, Gibson K, Cisneros J, Richards TJ, Yang Y, Becerril C, Yousem S, Herrera I, Ruiz V, Selman M, Kaminski N: Up-regulation and profibrotic role of osteopontin in human idiopathic pulmonary fibrosis. PLoS Med 2005;2:e251.
13 Zahradka P: Novel role for osteopontin in cardiac fibrosis. Circ Res 2008;102:270-272.

14 Mori R, Shaw TJ, Martin P: Molecular mechanisms linking wound inflammation and fibrosis: knockdown of osteopontin leads to rapid repair and reduced scarring. J Exp Med 2008;205:43-51.

15 Liaw L, Birk DE, Ballas CB, Whitsitt JS, Davidson JM, Hogan BL: Altered wound healing in mice lacking a functional osteopontin gene (spp1). J Clin Invest 1998;101:14681478.

16 Lenga Y, Koh A, Perera AS, McCulloch CA, Sodek J, Zohar R: Osteopontin expression is required for myofibroblast differentiation. Circ Res 2008;102:319-327.

17 Xie Y, Sakatsume M, Nishi S, Narita I, Arakawa M, Gejyo F: Expression, roles, receptors, and regulation of osteopontin in the kidney. Kidney Int 2001;60:1645-1657.

18 Mazzali M, Kipari T, Ophascharoensuk V, Wesson JA, Johnson R, Hughes J: Osteopontin - a molecule for all seasons. Q J Med 2002 95:3-13.

19 Yu XQ, Fan JM, Nikolic-Paterson DJ, Yang N, Mu W, Pichler R, Johnson RJ, Atkins RC, Lan HY: IL-1 up-regulates osteopontin expression in experimental crescentic glomerulonephritis in the rat. Am J Pathol 1999;154: 833-841.

20 Yu XQ, Nikolic-Paterson DJ, Mu W, Giachelli CM, Atkins RC, Johnson RJ, Lan HY: A functional role for osteopontin in experimental crescentic glomerulonephritis in the rat. Proc Assoc Am Phys 1998;110:50-64.

21 Bonvini JM, Schatzmann U, Beck-Schimmer B, Sun LK, Rittling SR, Denhardt DT, Le Hir M, Wüthrich RP: Lack of in vivo function of osteopontin in experimental anti-GBM nephritis. J Am Soc Nephrol 2000;11:16471655.

22 Yoo KH, Thornhill BA, Forbes MS, Coleman CM, Marcinko ES, Liaw L, Chevalier RL: Osteopontin regulates renal apoptosis and interstitial fibrosis in neonatal chronic unilateral ureteral obstruction. Kidney Int 2006; 70:1735-1741.

23 Wolak T, Kim H, Ren Y, Kim J, Vaziri ND, Nicholas SB: Osteopontin modulates angiotensin-II-induced inflammation, oxidative stress, and fibrosis of the kidney. Kidney Int 2009;76:32-43.
24 Susztak K, Böttinger E, Novetsky A, Liang D, Zhu Y, Ciccone E, Wu D, Dunn S, McCue P, Sharma K: Molecular profiling of diabetic mouse kidney reveals novel genes linked to glomerular disease. Diabetes 2004;53:784794.

25 Lorenzen J, Shah R, Biser A, Staicu SA, Niranjan T, Garcia AM, Gruenwald A, Thomas DB, Shatat IF, Supe K, Woroniecki RP, Susztak K: The role of osteopontin in the development of albuminuria. J Am Soc Nephrol 2008;19:884-890.

26 Wu J, Hicks J, Borillo J, Glass WF 2nd, Lou $\mathrm{YH}: \mathrm{CD}^{+} \mathrm{T}$ cells specific to a glomerular basement membrane antigen mediate glomerulonephritis. J Clin Invest 2002;109:517524.

27 Wu J, Hicks J, Borillo J, Glass WF 2nd, Lou YH: T cell epitope of glomerular basement membrane antigen induces severe glomerulonephritis. Kidney Int 2003;64:1292-1301.

-28 Da Costa CET, Annels NE, Faaij Claudia MJM, Forsyth RG, Hogendoorn PCW, Egeler RM: Presence of osteoclast-like multinucleated giant cells in the bone and non-ostotic lesions of Langerhans' cell histiocytosis. J Exp Med 2005;201:687-693.

29 Remuzzi A, Mazerska M, Gephardt GN, Novick AC, Brenner BM, Remuzzi G: Threedimensional analysis of glomerular morphology in patients with subtotal nephrectomy. Kidney Int 1995;48:155-162.

30 Lan HY, Yu XQ, Yang N, Nikolic-Paterson DJ, Mu W, Pichler R, Johnson RJ, Atkins RC: De novo glomerular osteopontin expression in rat crescentic glomerulonephritis. Kidney Int 1998;53:136-145.

31 Yu XQ, Wu LL, Huang XR, Yang N, Gilbert RE, Cooper ME, Johnson RJ, Lai KN, Lan HY: Osteopontin expression in progressive renal injury in remnant kidney: role of angiotensin II. Kidney Int 2000;58:1469-1480.

32 Hagood JS, Prabhakaran P, Kumbla P, Salazar L, MacEwen MW, Barker TH, Ortiz LA, Schoeb T, Siegal GP, Alexander CB, Pardo A, Selman M: Loss of fibroblast Thy-1 expression correlates with lung fibrogenesis. Am J Pathol 2005;167:365-379.

33 Mulder GD, Vande Berg JS: Cellular senescence and matrix metalloproteinase activity in chronic wounds. J Am Podiatr Med Assoc 2000;92:34-37. 